\title{
Mortality trends in a Cohort of Canadian Psoriatic Arthritis Patients
}

\author{
Boluwaji Ogunyemi \\ Memorial University of Newfoundland
}

\begin{abstract}
Methods: We reviewed retrospectively the charts of psoriatic arthritis patients who died from 1995-2010.We included 13 deceased patients with a psoriatic arthritis diagnosis and compared them with 140 patients living with psoriatic arthritis that attend the same clinic. The population was derived from a single academic rheumatologist's practice in St. John's, Newfoundland, Canada. Patients are seen at six-month intervals with a history and physical exam performed at each visit. Laboratory data was collected at each visit. Diagnosis of psoriatic arthritis is based on the CASPAR Classification and Diagnostic Criteria for Psoriatic Arthritis.

Results: The mean age of the 13 deceased patients was 62.9 years. Of these, $38.5 \%$ were female and $85.7 \%$ had an erythrocyte sedimentation rate greater than $15 \mathrm{~mm} /$ hour vs. $36.4 \%$ of patients living with psoriatic arthritis. Of deceased patients, $16 \%$ had dystrophic nail changes of vs. $59.6 \%$ of living patients. Health Assessment Questionnaire was found to show a significantly greater loss in function in deceased patients. (1.39 vs. $0.70, p=0.002$ ). Almost half of the deceased patients had used Prednisone (46.2\%) as opposed to $11.2 \%$ of living patients.

Conclusions: We realize that this study employs a small sample size. Increased ESR and Health Assessment Questionnaire score were found to be associated with mortality in psoriatic arthritis patients. Dystrophic nail changes were found to be protective for psoriatic arthritis patients.
\end{abstract}

Key Words: Psoriasis, Psoriatic Arthritis, Mortality, Arthritis Epidemiology

\section{INTRODUCTION}

Psoriatic arthritis (PsA) is often classified as a seronegative inflammatory spondylarthropathy and affects about $10 \%$ of individual affected with psoriasis. PsA occurs at a median of 10 years after the onset of psoriases but $15-20 \%$ of PsA patients will develop joint lesions before skin lesions due to psoriases appear (1).

Psoriatic arthritis was once thought to be a milder form of rheumatoid arthritis however many studies have subsequently established that PsA is often aggressive and is associated with various comorbidities and often takes a chronic, progressive course(2).The population of Newfoundland and Labrador, Canada lends itself to the study of PsA because of unique genetic characteristic in this population. Geographic isolation, relative genetic homogeneity and the founder effect lend this population with unique genetic distribution that proves useful in investigation diseases such as PsA compare to admixed Caucasian populations in the world. A significant association between the presence of PsA and a gene named CARD15 has been discovered in a 2003 study by Rahman et al. (OR=2.97, $p=0.0005)$ (3). There is a paucity of literature that investigates which factors affect mortality in psoriatic arthritis patients

Boluwaji Ogunyemi

4 Lewisporte Place, St. John's

Newfoundland and Labrador, Canada, A1E 5T3

Tel: 709.764.0350, Fax 709.745.2639

b.ogunyemi@mun.ca 
Table 1. Demographics and family history in deceased PSA vs early and established psa

\begin{tabular}{|l|l|l|}
\hline & Deceased PsA Patients $\mathbf{( n = 1 3 )}$ & $\begin{array}{l}\text { Early \& Established PsA Patients } \\
(\mathbf{n}=\mathbf{1 4 0})\end{array}$ \\
\hline Mean Age & 55.5 Years & 49.7 Years \\
\hline Sex & $61.5 \%$ Male & $47.2 \%$ Male \\
& $38.5 \%$ Female & $52.8 \%$ Female \\
\hline Family History of Psoriasis & $25 \%$ & $27.8 \%$ \\
\hline Family History of Arthritis & $38 \%$ & $55.3 \%$ \\
\hline Family History of Psoriatic Arthritis & $7.7 \%$ & $18.6 \%$ \\
\hline
\end{tabular}

in a Newfoundland and Labrador rheumatology practice.

Incidence of psoriatic arthritis has been estimated to be six per 100,000 adults aged 16 and over in Finland by Kaipiainen-Seppanen and Aho (4) with a mean age of diagnosis of 46.8 years of age. Shbeeb et al (5) conducted a study in Minnesota, USA and considered inflammatory

\section{METHODS}

We identified patients in a rheumatology practice in St. John's, Newfoundland \& Labrador, Canada with a definite diagnosis of PsA that have died in the period from January 1st 1999 to December 31st 2009. Similarly to Gladman and colleagues, $(11,13)$, the patients that compose both the deceased PsA patients and living patients in this study are treated in a single outpatient clinic.

Patients are seen at six-month intervals with a history and physical exam performed at each period. Further, laboratory data was collected at each visit. Diagnosis of psoriatic arthritis based on the CASPAR Classification and Diagnostic Criteria for Psoriatic Arthritis (14).

Similarly to a study by Khraishi and Murphy, (15) patient death will be ascertained by the following means: review of charts in the arthritis clinic, review of hospital records and death certificates. Further, local newspaper obituary section and contact with family members as well as family physicians will be employed to document patient death.

Basic demographic variables as well as clinical variables relevant to psoriatic arthritis such as nail involvement (pitting or onycholysis), family history of psoriasis, PsA or other arthropathy and presence and severity of psoriasis will be compared in the deceased psoriatic arthritis patients with patients living with psoriatic arthritis in that attended the same clinic. Health Assessment Questionnaire (HAQ) score gives a measure of how debilitating arthritis is in terms of reduced capacity to perform daily activities and will also be assessed. This tool has been validated in use with psoriatic arthritis patients. (16) HAQ is a patient self-assessment scale that ranges from a low of zero to a high of three for high self-perceived disability. In this study, we will investigate the Standard Disability Index portion of the HAQ.

Clinical signs related to psoriatic arthritis that will be evaluated include distal interphalyngeal joint (DIP) involvement, axial skeleton involvement, dystrophic nail changes (nail involvement), asymmetry of joint involvement, oligoarthritis, psoriasis and erythrocyte sedimentation rate. Asymmetry of joint involvement was defined as a difference of more than one in the number of affected joints between contralateral hands and feet. Oligoarthritis was defined as four or fewer joints displaying swelling or tenderness.

Medications that will be investigated include concomitant use of diabetes mellitus and hypertension medication as well as medication for depression or anxiety. The following disease-specific medicationswere investigated: methotrexate, sulfasalizine, prednisone, hydroxychloriquine, calcipotriol, (Dovonex), etanercept (Enbrel), infliximab (Remicade), and adalimumab (Humira). Disease-modifying anti-rheumatic drugs (DMARDS) included defined utilization of one or more of the following medications: methotrexate, sulfasalozine, hydroxychloroquin and plaquenil. The use of biologics includes the use of one or more of the following medications: etanercept, infliximab, and adalimumab.

\section{STATISTICAL METHODS}

Proportions for categorical variables will be reported as well as means and standard deviations doe continuous variables analyzed and compared between deceased patients that were diagnosed with PsA and patients living with PsA. Further, an Independent samples T-test will be employed to test 
Table 1. Demographics and family history in deceased PSA vs early and established psa

\begin{tabular}{|l|l|l|}
\hline Clinical Sign & Deceased PsA Patients $(\mathbf{n}=\mathbf{8})$ & $\begin{array}{l}\text { Early \& Established PsA Patients } \\
(\mathbf{n}=\mathbf{1 4 0})\end{array}$ \\
\hline DIP Involvement & $25 \%$ & $39.8 \%$ \\
\hline Axial Involvement & $13 \%$ & $19.8 \%$ \\
\hline Axial and Peripheral Involvement & $13 \%$ & $21.2 \%$ \\
\hline Nail Involvement* & $38 \%$ & $59.6 \%$ \\
\hline Asymmetry of Joints & $57 \%$ & 50.4 \\
\hline Oligoarthritis & $43 \%$ & $42.1 \%$ \\
\hline Erythrocyte Sedimentation Rate $>15^{*}$ & $63 \%$ & $36.4 \%$ \\
\hline
\end{tabular}

Asterisk $\left({ }^{*}\right)$ represents a statistically significant difference at $p=0.05$.

Erythrocyte sedimentation rate over 15 was significantly more prevalent in decreased PsA patients (63\%) than in early and established

for statistically significant differences in continuous variables between deceased patients with PsA and patients living with PsA.

The Chi-square statistic was used to detect associations between mortality and medication use, presence of comorbidities as well as clinical features of PsA. Fisher's exact test was employed in the case of low numbers. For all analyses, the minimum level of statistical significance, is 0.05 , determined a priori. All statistical analyses were performed using SPSS version 15.0 for Windows (SPSS Inc.).

\section{RESULTS}

There were statistically significant differences between prevalence of hematological comorbidities between deceased patients and patients living with PsA. ( $38 \%$ vs. $8.3 \%, p=0.033)$. Of these, the most common hematological comorbidity in both groups was anemia. In a review by Gabriel and Michaud, anemia was found to be a comorbid condition commonly found with Rheumatoid arthritis as well. Musculoskeletal comorbidies were very common in both deceased patients and patients living with PsA (50\% and 57.6\%).

Psoriasis is part of the criteria for diagnosing psoriatic arthritis $(83 \%$ of deceased patients and $95.1 \%$ of patients living with PsA).

Not surprisingly, there was a greater proportion of deceased psoriatic arthritis patients with malignancy than those with early and established diseased. Specifically, Bowel cancer ( $13 \%$ vs $0.7 \%)$, breast cancer $(0 \%$ vs $1.4 \%)$, lymphoma ( $25 \%$ vs $0.7 \%)$, other cancers (13\% vs $5.6 \%$ ) and total proportion of patients with a malignancy (38\% vs $7.6 \%)$. Of those, only the total number of malignancies was statistically significant at $a=0.05$.
When comparing medications between patients with early and established disease, the only medications that were shown to be statistically significantly higher in use in early and established patients was Prednisone. $(p=0.011)$. Other mediations investigated including methotrexzte, Enbrel, Remicade, Sulfasalazine, Hydroxychloroin, Humira, were not found to be used in a different amount between the early \& established and mortality.

Neither a family history of psoriasis $(p=$ $0.864)$, psoriatic arthritis $(p=0.180)$ or other type of arthritis $(p=0.464)$. In terms of clinical features characteristic for psoriatic arthritis, there was a statistically significant increase in the proportion of deceased patient with an ESR greater than 15 $\mathrm{mm} /$ hour in deceased patients and a statistically significant decreases proportion of nail involvement in deceased patients.

Other clinical signs of psoriatic arthritis were not found to have a statistically significant prevalence in the two groups investigated including axial involvement $(p=0.611)$, distal interphalangeal joint involvement $(p=0.482)$, asymmetrical joint distribution $(p=0.699)$, oligoarthritis $(p=0.441)$.

\section{DISCUSSION}

Though cardiovascular comorbidities have been specifically implicated in rheumatoid arthritis patients and indeed a major source of their increased mortality, prevalence of cardiovascular comorbidities is not significantly elevated in either group of PsA patients in this study.

When we compared the following continuous variables ages, age at diagnosis of PsA and diagnosis of psoriasis, most recent Health Assessment Questionnaire score (HAQ), systolic 
Table 3. Comorbidities in deceased versus early and established Psoriatic Arthritis

\begin{tabular}{|c|c|c|}
\hline Comorbidity & Deceased PsA Patients $(n=13)$ & $\begin{array}{l}\text { Early \& Established PsA Patients } \\
(n=140)\end{array}$ \\
\hline Hypertension & $38 \%$ & $32.6 \%$ \\
\hline Psoriasis & $83 \%$ & $95.1 \%$ \\
\hline Coronary Heart Disease & $13 \%$ & $4.2 \%$ \\
\hline Musculoskeletal History & $50 \%$ & $57.6 \%$ \\
\hline Gastrointestinal History & $38 \%$ & $45.8 \%$ \\
\hline Angina & $0 \%$ & $0 \%$ \\
\hline TIA & $0 \%$ & $0.7 \%$ \\
\hline Respiratory System History & $13 \%$ & $20.8 \%$ \\
\hline Obesity & $38 \%$ & $47.2 \%$ \\
\hline Thyroid Disease & $13 \%$ & $4.2 \%$ \\
\hline Peripheral Vascular Disease & $0 \%$ & $11.8 \%$ \\
\hline Hepatobiliary System History & $25 \%$ & $16.0 \%$ \\
\hline Peptic Ulcer & $13 \%$ & $4.9 \%$ \\
\hline Hematological-Lymphatic History* & $38 \%$ & $8.3 \%$ \\
\hline $\begin{array}{l}\text { Cardiovascular System History } \\
\text { No cardiovascular comorbidities }\end{array}$ & $87 \%$ & $91.5 \%$ \\
\hline $\begin{array}{l}\text { Cardiovascular System History } \\
\text { One cardiovascular comorbidities }\end{array}$ & $0 \%$ & $6.4 \%$ \\
\hline $\begin{array}{l}\text { Cardiovascular System History } \\
\text { Two or more cardiovascular comorbidities }\end{array}$ & $13 \%$ & $2.1 \%$ \\
\hline
\end{tabular}

Asterisk $\left({ }^{*}\right)$ represents a statistically significant difference at $p=0.05$.

blood pressure, erythrocyte sedimentation rate (ESR) and total number of tender joints between deceased PsA patients to those defined with early or established PsA, we found HAQ score and ESR to have statistically significant associations with mortality. The deceased patients with PsA had a significantly higher $\mathrm{HAQ}$ score than patients still living with PsA: 1.47 vs. $0.70(p=0.014)$. The eight equally-weighted areas of the HAQ are difficulty in everyday activities, arising, dressing \& grooming, eating, grip, performing activities related to hygiene, reach and walking.

Since ESR is a non-specific marker for inflammation, ESR may be related to severity of this seronegative inflammatory condition. The mean ESR of $84.6 \mathrm{~mm} /$ hour for deceased PsA patients vs. $20.6 \mathrm{~mm} /$ jour for patients living with PsA is significant. $(p=0.021)$ This is similar to a study by Gladman et al, (11) which found that ESR $>15 \mathrm{~mm} /$ hour had value in predicting mortality of PsA patients. When ESR was categorized as such in this study, there was a significant association between mortality and ESR status in PsA patients. $(p=0.013)$

Beside erythrocyte sedimentation rate, presence of nail lesions (pitting or onchylysis) seems to be the only other statistically significant clinical variable. Similarly to Gladman et al (2), we discovered that a higher proportion of patients living with PsA that deceased patients that were diagnosed with PsA had nail involvement $(59.6 \%$ vs. $38 \%, p=0.013$ ), this acts as a protective factor against mortality in PsA patients. This may be because the presence of nail involvement may cause patients to seek treatment earlier in the course of their disease.

There was no statistically significant difference in the proportions of prescribed nonPsA (concomitant) medication between deceased patients and those living with PsA. Methotrexate is the most commonly-prescribed DMARD in both groups and was prescribed to $38 \%$ of deceased PsA patients and $28 \%$ of patients with established PsA (Table 4). Prednisone was found to be 
Table 4. Quantitative data in deceased PSA patients vs early \& established PSA

\begin{tabular}{|l|l|l|}
\hline Quantitative Variable & Deceased PsA Patients $\mathbf{( n = 1 3 )}$ & $\begin{array}{l}\text { Early \& Established PsA Patients } \\
(\mathbf{n = 1 4 0})\end{array}$ \\
\hline $\begin{array}{l}\text { Age as of December 31, 2009 or } \\
\text { Age at Death }\end{array}$ & 55.50 Years & 49.67 Years \\
\hline Age of Diagnosis Psoriasis & 31.75 Years & 35.70 Years \\
\hline Age of Diagnosis Psoriatic Arthritis & 47.00 Years & 44.78 Years \\
\hline Systolic Blood Pressure & $134.14 \mathrm{mmHg}$ & $126.58 \mathrm{mmHg}$ \\
\hline Erythrocyte Sedimentation Rate $*$ & $84.60 \mathrm{~mm} / \mathrm{hour}$ & $20.62 \mathrm{~mm} / \mathrm{hour}$ \\
\hline Total Tender Joints & 5.00 & 7.42 \\
\hline Health Assessment Questionnaire* & 1.4725 & .6968 \\
\hline
\end{tabular}

Asterisk $\left({ }^{*}\right)$ represents a statistically significant difference at $p=0.05$.

statistically associated with morality in PsA patients $(p=0.011)$ as $50 \%$ of deceased patients had been prescribed Prednisone while only $11.2 \%$ of patients living with PsA enrolled at the same rheumatology clinic were using Prednisone. Further, use of any DMARDS was found be to significantly associated with mortality in PsA patients $(p=0.007)$. When we removed patients that had been treated with prednisone from our analysis, we found that results were not significant $p=0.121$.

For each of the individual malignancies of breast cancer, bowel cancer and lymphoma, there was no statistically significant difference between the prevalence of these comorbidities in the two groups. However, when the proportion (Table 4) of patients with any type of malignancy was compared between patients with early and established PsA and deceased patients with PsA, we found that a significantly greater proportion of deceased patients suffer from at least one malignancy (38\% vs. $7.6 \%, p=0.027$ ) We found no statistically significant relationship between rheumatoid factor and positive history of cardiovascular events in PsA patients.

\section{CONCLUSION}

The presence of at least one malignancy was found to be related to increased likelihood of mortality in PsA patients though a relationship between particular cancers and mortality could not be ascertained. Moreover, use of DMARDS was also found to $b$ statistically associated with mortality in PsA patients. Specifically, the use of Prednisone as found to be associated with mortality in PsA patients. Further, increased ESR and the absence of dystrophic nail changes are associated with mortality in PsA patients.

\section{LIMITATIONS}

We understand that it may be difficult to apply conclusions for these results to all who suffer from psoriatic arthritis since the information is relevant to individuals that have been treated at a clinic; milder cases of psoriatic arthritis may be missed.

Further, the relatively small sample size of deceased psoriatic arthritis patients makes it difficult to properly interpret results from this group.

Since all patients from this study are seen in an outpatient clinic, we the results may show greater overall morbidity and mortality that in PsA in the general population that may be so mild as to go undetected or not require medical attention.

As a cross-sectional study, this study does not support inferences in causality. That is, though we can say that specific comorbidities and medications are associated with mortality in PsA patients, we cannot ascertain whether or not these characteristics cause an increased risk of death from PsA or whether these patients were sicker on the whole and therefore needed numerous diseasespecific drugs.

\section{ACKNOWLEDGMENTS}

I would like to thank the Graduate program in Clinical Epidemiology and the Faculty of Medicine at Memorial University of Newfoundland.

I would also like to thank Research and Graduate Studies in the Faculty of Medicine and the Summer Undergraduate Research Award Program - Medical who have helped to make this project possible and their support is appreciated. 


\section{REFERENCES}

1. Taylor, W., Gladman, D., Helliwell, P., Marchesoni, A., Mease, P., Mielants, H. Classification criteria for psoriatic arthritis: Development of new criteria from a large international study. Arthritis and Rheumatism.. 2006; Aug;54(8):2665-73.

2. Gabriel, S. And Michaud, K: Epidemiological studies in incidence, prevalence, mortality, and comorbidity of the rheumatic diseases. Arthritis Research \& Therapy 2009; $11: 229$

3. Rahman, P., Jones, A., Curtis, J., Bartlett, S., Peddle, L., Fernandez, B., and Freimer, N.The Newfoundland population: a unique resource for genetic investigation of complex diseases. Human Molecular Genetics. 2003;12 Review Issue 2 R167-R172

4. Shbeeb M, Uramoto KM, Gibson LE, O'Fallon WM, Gabirel SE: The epidemiology of psoriatic arthritis in Olmsted County, Minnesota, USA. 198201991. Journal of Rheumatology 2000; 27: 1247-50.

5. Wilson FC, Icen M, Crowson CS, McEvoy MT, Gabriel SE and Kremers, HM: Time trends in epidemiology and characteristics of psoriatic arthritis over three decades: a population-based study. Journal of Rheumatology 2009; 36:361-7

6. Wong K, Gladman DD, Husted J, Long JA, and Farewell VT: Mortality studies in psoriatic arthritis: results from a single outpatient clinic I. Causes and risk of death. Arthritis Rheum 1997, 40: 1868-1872.

7. Mallbris L, Akre O, Granath F, Yin L, Lindelof B, Ekbom A, and Stahle-Backdahl M. Increased risk for cardiovascular mortality in psoriasis inpatients but not in outpatients. European Jourbnal of Epidemiology. 2004;19(3):225-30.

8. Ali Y, Tom BD, Schentag CT, Farewell VT, and Gladman DD. Improved survival in psoriatic arthritis with calendar time. Arthritis and Rheumatism. 2007 Aug;56(8):2708-14.

9. Wilson FC, Icen M, Crowson CS, McEvoy MT, Gabriel SE. Maradit Kremers: Time trends in epidemiology and characteristics of psoriatic arthritis over 3 decades: a population-based study. Journal of Rheumatology 2009; 36:361-7.

10. Maradit-Kremers H, Nicola, PJ, Crowson, CS, Ballman KV, Gabriel SE: Cardiovascular death in rheumatoid arthritis: a population-based study. Arthritis Rheum 2005; 52: 722-3.

11. Gladman DD, Farewell VT, Wong K, Husted J: Mortality Studies in psoriatic arthritis: results from a single outpatient center, II. Prognostic indicators for death. Arthritis Rheum 1998, 41: 1103-10.

12. Kammer GM, Soter NA, Gibson DJ, Schur PH. Psoriatic arthritis: a clinical, immunological and HLA study of 100 patients. Semin Arthritis Rheum 1979; 9:75-97

13. Gladman, D. Mortality in Psoriatic Arthritis. Clinical Experimental Rheumatology 2008; 26 (Suppl. 51) S62-S65

14. Helliwell, PS and Taylor, WJ (2005). Classification and diagnostic criteria for psoriatic arthritis. Ann Rheum Dis. 2005; Mar;64 Suppl 2:ii3-8.

15. Khraishi, M. And Murphy, M. Mortality trends in a cohort of Canadian Rheumatoid Arthritis Patients. 2003; European League Against Rheumatism (EULAR).

16. Husted JA, Gladman DD, Long JA, Farewell VT. A modified version of the Health Assessment Questionnaire (HAQ) for psoriatic arthritis. Clinical and Experimental Rheumatology 1995 Jul-Aug;13(4):439-43. 